\title{
Review Article \\ The Role of Transforming Growth Factor-Beta in Diabetic Nephropathy
}

\author{
Karina Braga Gomes, ${ }^{1,2,3}$ Kathryna Fontana Rodrigues, ${ }^{1,2}$ and Ana Paula Fernandes ${ }^{1,2}$ \\ ${ }^{1}$ Instituto de Ciências Biológicas, Universidade Federal de Minas Gerais, 31270-901 Belo Horizonte, MG, Brazil \\ ${ }^{2}$ Faculdade de Farmácia, Universidade Federal de Minas Gerais, 31270-901 Belo Horizonte, MG, Brazil \\ ${ }^{3}$ Departamento de Análises Clínicas e Toxicológicas, Faculdade de Farmácia, Universidade Federal de Minas Gerais, \\ Avenida Antônio Carlos 6627, Pampulha, 31270-901 Belo Horizonte, MG, Brazil \\ Correspondence should be addressed to Karina Braga Gomes; karinabgb@gmail.com
}

Received 5 October 2013; Revised 16 November 2013; Accepted 4 December 2013; Published 28 January 2014

Academic Editor: George N. Goulielmos

Copyright (C) 2014 Karina Braga Gomes et al. This is an open access article distributed under the Creative Commons Attribution License, which permits unrestricted use, distribution, and reproduction in any medium, provided the original work is properly cited.

\begin{abstract}
Several studies have demonstrated that chronic and low-grade inflammation is closely linked to type 2 diabetes mellitus. The associated mechanisms are related to synthesis and release of proinflammatory and anti-inflammatory cytokines, mainly by the adipose tissue. Moreover, there are evidences that cytokines and adhesion molecules are important for development of diabetic nephropathy. Among the cytokines associated with inflammatory responses in type 2 diabetes mellitus, the transforming growth factor- $\beta$ (TGF- $\beta$ ) has been recognized as a central player in the diabetic nephropathy being involved in the development of glomerulosclerosis and interstitial fibrosis, as observed in the course of end-stage renal disease. Although TGF- $\beta 1$ is classically an anti-inflammatory immune mediator it has been shown that in the presence of IL-6, which increases before the onset of T2D, TGF- $\beta 1$ favors the differentiation of T helper 17 (Th17) cells that are activated in many pro-inflammatory conditions. Since TGF- $\beta 1$ mRNA and consequently serum TGF- $\beta 1$ levels are under genetic control, this review aims to discuss the relationship of TGF- $\beta 1$ levels and polymorphisms in the development of nephropathy in type 2 diabetes mellitus.
\end{abstract}

\section{Introduction}

Type 2 diabetes mellitus (T2D) is one of the most important global public health problems. T2D prevalence is rapidly increasing and it is considered an epidemic disorder worldwide. Moreover, its prevalence will continue to rise in the next 20 years, with special concerns regarding incidence raises in children and adolescents [1].

In the last years, several studies have demonstrated that chronic and low-grade inflammation is closely involved in the T2D development. The mechanisms are associated with synthesis and release of proinflammatory cytokines, mainly by the adipose tissue [2]. Cytokines are a group of pharmacologically active polypeptides included in several classes: interleukins (IL), tumor necrosis factors (TNF), interferons, colony-stimulating factors (CSF), transforming growth factors (TGF), and chemokines, which participate as humoral mediators and coordinate a network regulating inflammatory immune responses. Furthermore, they exert important pleiotropic actions as cardinal effectors of injury [2]. Several studies showed that the activation of the innate immune system is also involved in T2D. The innate immune system modulates the effects of many factors including genes, cytokine levels, and metabolic events associated with insulin resistance $[3,4]$.

TGF- $\beta 1$ is an anti-inflammatory immune mediator which inhibits or reverses the activation of macrophages by interfering with signaling via toll-like receptor-dependent pathways. Herder et al. [5] reported that high levels of TGF- $\beta 1$ were associated with T2D and elevated IL-1Ra concentration, whose increase precedes the development of T2D. Therefore, pro- and anti-inflammatory mediators are present in T2D and TGF- $\beta 1$ may act in both sides.

The incidence of end-stage renal disease (ESRD) is increasing worldwide in T2D due to progressive injury in the glomeruli or within the tubule-interstitium [6]. Diabetic 
nephropathy is considered the most common cause of ESRD and inflammatory process characterized by macrophage infiltration is observed during all stages of renal involvement [7].

There are evidences that proinflammatory cytokines and adhesion molecules are important in the development of diabetic nephropathy [8]. Cross-sectional studies in T2D have shown that inflammatory biomarker levels as C-reactive protein (CRP) and adhesion molecules as E-selectin are increased in subjects with nephropathy $[9,10]$. Moreover, Astrup et al. [11] showed that sVCAM-1 was independently predictive of progression of renal disease in diabetics over a 10 -year period of observation.

Over the past several years, transforming growth factor$\beta$ (TGF- $\beta$ ) has been recognized as a central player in the diabetic nephropathy. In this way, the present review aims to discuss the relationship between TGF- $\beta$, especially its polymorphisms, and the development of nephropathy in T2D.

\section{TGF- $\beta$ Complex and How It Is Linked to Diabetic Nephropathy}

The TGF- $\beta$ family is composed of 33 cytokines and they exert their effects by forming complexes of type I and II serine/threonine kinase receptor [12]. The type II receptor is phosphorylated and activates the type I receptor, which further phosphorylates the Smad family. The Smad family comprises three subfamilies: R-Smads (Smad1-3, Smad5, and Smad8), a common-mediator Co-Smad (Smad4) and the inhibitors I-Smads (Smad6 and Smad7). The R-Smads are phosphorylated by the Type I receptors, form a complex with Smad4 and translocate to the nucleous. There, this complex regulates the gene expression associated with growth arrest, immune system control, angiogenesis, cell differentiation, and epithelial-mesenchymal transition (EMT), by altering nucleosome structure and thereby remodeling the chromatin template, mediated by the interaction between the $\mathrm{MH} 2$ domain and R-Smads [12-14].

Epithelial-mesenchymal transition (EMT) is a cellular process, wherein adherent cells disintegrate their intercellular contacts, organize their motility complex, and shift to new locations during the formation of fibrotic tissues. TGF- $\beta$ is considered a main inducer of EMT in epithelial tissues through secretion of chemokines, platelet-derived growth factor-A (PDGF-A), and PDGF-G receptors that promote EMT. EMT apparently is the main process involved in de novo expression of alpha-smooth actin ( $\alpha$-SMA), which characterizes the interstitial myofibroblasts (IM), in destruction of Ecadherin and fibre formation. Both tubular and glomerular epithelial cells are able to trans-differentiate into IM. The IM constitute a substantial source of tubulointerstitial extracellular matrix (ECM) and are considered one of the best indicators of disease progression [14].

Nephropathy is characterized by renal hypertrophy and deposition of ECM that leads to glomerulosclerosis. Consequently, albuminuria, high blood pressure, a progressive decrease in glomerular filtration rate (GFR), uraemia, and end-stage renal disease (ESRD) are the intermediate and long-term outcomes of the disease [8].
The hyperglycemia commonly observed in patients with T2D is associated with upregulation of glucose transport-1 which leads to TGF- $\beta$ overexpression by mesangial tubular cells or infiltrating renal cells $[3,15,16]$. In addition, increased intraglomerular pressure, mesangial cell stretch, activation of renin-angiotensin system, reactive oxidant species (ROS) and advanced glycation end products (AGEs) have been shown to induce TGF- $\beta$ production in the kidney or in cultured mesangial or tubular cells. The TGF- $\beta$ acts through autocrine and paracrine pathways and stimulates its signaling receptors and ECM synthesis (Figure 1(a)) [17, 18].

Clinical evidences suggest that the response triggered by TGF- $\beta$ involves a final pathway leading to the development of glomerulosclerosis and interstitial fibrosis in the course of ESRD [19]. TGF- $\beta$ has been considered as a major cytokine involved in the regulation of ECM synthesis, stimulating the production of its components, including collagen, fibronectin and proteoglycans and blocking matrix degradation [20]. These events induce the increase of vascular permeability, and finally permit loss of low-molecular-weight albumin [15]. It is important to highlight that promoter areas of collagen genes (COL1A2, COL3A1l, OL5A1, COL6A1, COL6A3, and COL2A1) have Smad3 binding sequences, contributing to the TGF- $\beta$ induced ECM expression [14].

Rivarola et al. [20] evaluated TGF- $\beta$ in urine samples from healthy individuals and patients with T2D. They observed an increase in the rate of urinary TGF- $\beta$ excretion in patients with nephropathy when compared to normal individuals. They also showed a positive correlation between the rate of urinary TGF- $\beta$ excretion and proteinuria, one of the parameters of renal disease progression.

The TGF- $\beta$ presents a complex involvement with the pathophysiological progression of the renal disease regarding the inflammatory process. TGF- $\beta$ is involved in the resolution of renal injury including induction of glomerular capillary formation, apoptosis of mesangial cells, and inhibition of inflammatory cytokines production, such as interleukin$1 \beta$ and TNF- $\alpha$ [14]. In addition, TGF- $\beta$ inhibits $\mathrm{T}$ cell proliferation and leucocyte infiltration by blocking monocyte chemotactic protein-1 (MCP-1) and adhesion molecules expression [14].

The TGF- $\beta 1$ converts naive $\mathrm{T}$ cells into regulatory $\mathrm{T}$ cells. However, in the presence of IL6, a cytokine commonly increased in T2D individuals, TGF- $\beta 1$ promotes the differentiation of naive $\mathrm{T}$ lymphocytes into proinflammatory $\mathrm{T}$ helper that produces IL17, a process that is amplified by IL- $1 \beta$ and TNF- $\alpha$ [21]. IL17 promotes granulopoiesis and neutrophil accumulation, playing an active role in inflammatory diseases $[6,22]$. Okamoto el al. [23] identified a nuclear IkappaB family member, IkappaBzeta $(\mathrm{I} \kappa \mathrm{B} \zeta)$, as a transcription factor required for Th17 development. The ectopic expression of $\mathrm{I} \kappa \mathrm{B} \zeta$ in naive $\mathrm{CD}^{+}{ }^{+} \mathrm{T}$ cells potently induces Th17 development, even in the absence of IL- 6 and TGF- $\beta$ (Figure 1(b)).

Border et al. [24] reported that the administration of a TGF- $\beta$ inhibitor prevents the increased production of matrix proteins by glomerular cells and blocks ECM accumulation in an animal model of glomerulonephritis. Moreover, it has been shown that transfection of the TGF- $\beta$ gene into the rat kidney leads to high TGF- $\beta$ levels in glomeruli associated 


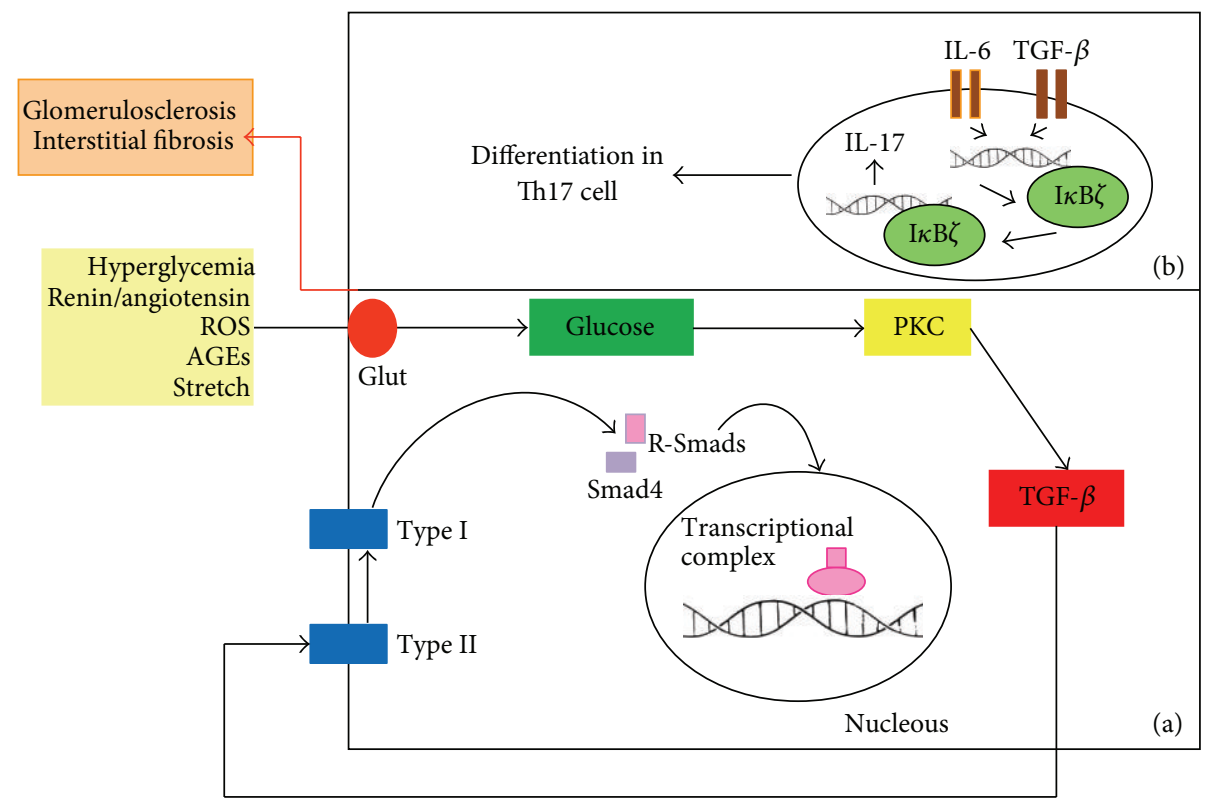

FIGURE 1: Activation of TGF- $\beta$ synthesis and its role in proinflammatory mechanisms in T2D nephropathy. (a) Increased extracellular glucose levels, mesangial cell stretch, activation of renin-angiotensin system, reactive oxidant species (ROS), and advanced glycation end products (AGEs) activate TGF- $\beta$ synthesis via protein kinase C. TGF- $\beta$ stimulates its own pathway through autocrine or paracrine action. TGF- $\beta$ assembles a receptor complex that activates Smads that regulate nuclear transcription. (b) TGF- $\beta 1$ and IL-6 promote the differentiation of naive T lymphocytes into proinflammatory T helper that produces IL17 through the transcription factor I $\kappa \mathrm{B} \zeta$. The outcomes of these processes are glomerulosclerosis and interstitial fibrosis.

with a rapid development of glomerulosclerosis [20, 25]. In addition, Niemir et al. [26] showed that anti-TGF- $\beta$ antibody prevented the increases in collagen and fibronectin expression and mesangial matrix expansion in mice with type 2 diabetes-like disease.

These data have led to the hypothesis that TGF- $\beta$ inhibitors may be renoprotective in diabetic patients with nephropathy [27]. Ohtomo et al. [28] have examined the possible renoprotective properties of the thiazolidinedione pioglitazone in rats, as a model of T2D with nephropathy. They reported that pioglitazone prevented proteinuria, limited glomerulosclerosis/tubulointerstitial fibrosis, normalized the insulin levels, and decreased the intrarenal TGF$\beta$ mRNA expression. In agreement, in a streptozotocinexperimental model of induced diabetes, pioglitazone suppressed the renal expression of TGF- $\beta$, reduced urinary albumin excretion, and ameliorated glomerular injury in the other study applied [29]. Therefore, local expression TGF$\beta$ mRNA seems to be an important component of diabetic nephropathy.

\section{TGF- $\beta 1$ Gene Polymorphisms and Diabetic Nephropathy}

TGF- $\beta 1$ precursor gene contains 7 exons and 6 very large introns in 19q13.1-q13.3. More than 10 polymorphic loci are presently known, distributed in exons, introns, and the 50flanking region [30, 31]. The deduced precursor protein contains 391 amino acids, of which the C-terminal 112 amino acids constitute the mature protein. An arg-arg dipeptide precedes the proteolytic cleavage site [32]. Some studies observing large interindividual differences concluded that TGF- $\beta 1$ mRNA and consequently serum TGF- $\beta 1$ levels are under genetic control [33,34].

Akai et al. [35] investigated the association of TGF$\beta 1$ T29C single nucleotide polymorphism (SNP) and the progression of diabetic nephropathy. Forty Japanese patients with T2D for more than 10 years were enrolled in the study. The patients were classified into three groups according to genotype: TT, TC, CC, and the grade of nephropathy was determined using the urinary excretion of albumin. Patients with urinary albumin excretion less than $30 \mathrm{mg} / \mathrm{d}$ were defined as normoalbuminuric, with urinary albumin excretion between 30 and $300 \mathrm{mg} / \mathrm{d}$ were defined as microalbuminuric, and patients with urinary albumin excretion greater than $300 \mathrm{mg} / \mathrm{d}$ were defined as albuminuric patients. The authors did not identify an association between the three genotypes and the severity of diabetic nephropathy. However, patients with TT genotype presented a tendency to higher rate of progression of nephropathy. Some limitations with this study, as pointed out by authors, include a small sample size which may not represent the entire diabetic population and secondly the use of urinary albumin excretion as a marker for progression of diabetic nephropathy, which is affected by several factors, other than diabetic nephropathy.

In order to evaluate the relationship between the TGF- $\beta 1$ T869C (Leu10Pro, codon 10) polymorphism and nephropathy, Wong et al. [36] studied 123 Chinese patients with T2D. The patients that developed nephropathy (micro or 
macroalbuminuria, with or without renal impairment) were compared with patients without renal alterations. The frequency of CC/CT genotypes was higher in the group with nephropathy and independently associated with this complication. Furthermore, patients with CC/CT genotypes and nephropathy presented worse renal function and increased risk for macroalbuminuria.

In a study conducted with individuals from Poland, Buraczynska et al. [37] investigated the polymorphisms T869C and C-509T of the TGF- $\beta 1$ gene in 503 patients and 400 healthy subjects. Only a significantly increased frequency of the $\mathrm{C}$ allele and CC genotype of the T869C polymorphism was observed in patients with nephropathy, suggesting the association of this polymorphism with an increased risk of T2D renal complication. Similar results were observed by Mou et al. [38] that investigated the T869C polymorphism in 180 T2D Chinese patients with nephropathy in stages III, IV, and $\mathrm{V}$, besides 180 patients without this complication. They also reported that $\mathrm{CC} / \mathrm{CT}$ genotypes were associated with an increased risk of worsening renal function.

Babel et al. [39] also studied the polymorphisms T869C and C915G (Arg25Pro, codon 25) in 118 German healthy donors and 44 hemodialysis patients with diabetic nephropathy. T869C polymorphism was associated with nephropathy, but no significant association was observed for C915G.

Valladares-Salgado et al. [40] discussed that hypercholesterolaemia and hypertriglyceridaemia aggravate renal injury not only by podocyte damage, fibrogenic activity, and renal inflammation but also by downregulation of renal metalloproteinases, thereby dulling extracellular matrix degradation. They observed that total cholesterol levels were associated with the T869C polymorphism in TGF$\beta 1$ gene. Total cholesterol levels were significantly higher in CC + CT genotypes, as compared to the TT genotype. In addition, significantly higher triglyceride concentrations were observed in CC + CT genotypes in comparison to the TT genotype. The study was conducted with 228 T2D Mexican patients with nephropathy and 192 patients without nephropathy. One of the proposed pathways linking hypercholesterolaemia and TGF- $\beta 1$ is that Ox-LDL stimulates its receptor, leading to glomerular injury by inducing formation of foam cells which, in turn, are associated with later glomerulosclerosis and interstitial injury [40, 41]. Also, hypercholesterolaemia, high TGF- $\beta 1$ levels, and anti-OxLDL result in activation of the innate immune response, inflammation, and fibrosis $[40,42]$. Besides these results, the authors also showed an association between T869C and C915G polymorphisms in TGF- $\beta 1$ gene, but not G$800 \mathrm{~A}$ in the promoter region, with nephropathy in this population.

The association between TGF- $\beta 1$ polymorphisms and diabetic nephropathy is still contradictory. Ahluwalia et al. [43] investigated the frequency of the polymorphisms Tyr81His (T>C), a functional SNP in the Exon 2, and T869C in diabetic patients with nephropathy $(n=336)$ and diabetic patients without nephropathy group $(n=397)$ from North and South India. The mean duration of T2D was similar in the two groups. The first SNP was found to be monomorphic in their population and was further not analyzed. The frequency of the minor allele of the T869C polymorphism was similar in cases and controls.

Likewise, Prasad et al. [44], in a case-control study including 196 cases and 225 controls of diabetic nephropathy from India, evaluated five SNPs in TGF- $\beta 1$ gene: G-800A, C-509 T, Arg25Pro, Tyr81His and Thr263Ile. Tyr81His, and Thr263Ile were monomorphic and Arg25Pro was a minor variant with frequency of variant allele $=0.01$. For the polymorphisms $\mathrm{G}-$ 800A and C-509T no significant association was observed with case group. The observed heterogeneity in these results when compared to other studies could be due to ethnic differences and small sample size.

A systematic review was conducted by Jia et al. [45] including 6 eligible case-control (1 study on Caucasian population, 1 on a mixed population, and 4 on Asian population3 Chinese, 1 Indian) that evaluated the association between the TGF- $\beta 1$ T869C polymorphism and diabetic nephropathy. As a result, significant increased risks for nephropathy were observed under allelic, additive, dominant and recessive genetic models. When confined to Asian T2D patients, significant association was detected only under allelic, additive and recessive model.

Recently, El-Sherbini et al. [3] conducted a study including 99 Egyptian patients with T2D categorized into 50 patients without diabetic nephropathy and 49 patients with diabetic nephropathy. Furthermore, 98 unrelated healthy age- and sex-matched nondiabetic, nonhypertensive subjects living in the same geographical area and having the same ethnic origin as patients were recruited as healthy controls. They investigated the possible role of the functional polymorphisms T869C and C915G. The last polymorphism did not show association with T2D or nephropathy. However, T2D patients had increased frequency of 869 CC and TC genotypes. The frequencies of $\mathrm{C}$ alleles were also higher among T2D patients than controls. The haplotype analysis also showed a significant association of TGF-1 which an excess of haplotypes CC and CG in T2D patients when compared to control group. Besides, increased frequency of TC genotype and absence of CC genotype in patients with nephropathy were reported.

In spite of the evidence that these TGF- $\beta 1$ polymorphisms, in particular T869C, are implicated in diabetic nephropathy, their role in determining TGF- $\beta 1$ levels or functional alterations is still largely unknown. Furthermore, the controversial results observed in these studies could be explained by different genetic background of the studied populations and discrepancies in nephropathy criteria of diagnosis, besides the molecular methods of genotyping.

\section{Future Prospects}

Some recent hypotheses should be further investigated in order to comprehend the complete role of TGF- $\beta$ in diabetic nephropathy etiology. For example, experimental models indicate increased expression of mitochondrial protein induced in high glucose 1 (IHG-1) which is a critical component of fibrogenesis and amplifies TGF- $\beta 1$ signalling [46]. Furthermore, blood glucose fluctuation can accelerate 
the trend of kidney fibrosis in diabetic mice by increasing collagen production and inhibiting collagen degradation, mediated by TGF- $\beta /$ smad signaling pathways [47].

Therefore, anti-TGF- $\beta$ antibody or TGF- $\beta$ inhibitors therapy may provide a renoprotective effect in diabetic patients with nephropathy. Indeed, the inhibition of the expression of microRNA-192 (miRNA-192), which is induced by TGF- $\beta$ and is associated with increased collagen expression, could be pointed as a future therapeutic target [17].

\section{Conclusion}

This review highlights the importance of TGF- $\beta$ in the development of T2D, the associated inflammatory responses, and progression of nephropathy. Besides, it reviews the evidence that TGF- $\beta$ inhibitors may have renal-protective effects in diabetic nephropathy. Among the TGF- $\beta 1$ gene polymorphisms so far investigated, T869C seems to be associated with diabetic nephropathy in some populations, although the phenotypic effects of these polymorphisms in TGF- $\beta$ levels or functionality are largely unknown. An additional limitation of all these studies is the small sample size. Therefore, confirmatory larger studies, including individuals of different ethnic origins, are still lacking. Further analyses of other genes or other TGF- $\beta 1$ polymorphisms are also warranted and may contribute to better elucidate the role TGF- $\beta 1$ gene and inflammatory responses in development a progression to complications in T2D.

\section{Conflict of Interests}

The authors declare that there is no conflict of interests regarding the publication of this paper.

\section{Acknowledgments}

The authors thank Conselho Nacional de Desenvolvimento Científico e Tecnológico (CNPq) and Fundação de Amparo à Pesquisa do Estado de Minas Gerais (FAPEMIG) for the financial support.

\section{References}

[1] J. F. Navarro and C. Mora, "Diabetes, inflammation, proinflammatory cytokines, and diabetic nephropathy," TheScientificWorldJOURNAL, vol. 6, pp. 908-917, 2006.

[2] J. F. Navarro-González and C. Mora-Fernández, "The role of inflammatory cytokines in diabetic nephropathy," Journal of the American Society of Nephrology, vol. 19, no. 3, pp. 433-442, 2008.

[3] S. M. El-Sherbini, S. M. Shahen, Y. M. Mosaad, M. S. Abdelgawad, and R. M. Talaat, "Gene polymorphism of transforming growth factor- $\beta 1$ in Egyptian patients with type 2 diabetes and diabetic nephropathy," Acta Biochimica et Biophysica Sinica, vol. 45, no. 4, pp. 330-338, 2013.

[4] J. C. Pickup, G. C. Chusney, S. M. Thomas, and D. Burt, "Is type II diabetes mellitus a disease of the innate immune system?" Life Sciences, vol. 67, no. 3, pp. 291-300, 2000.

[5] C. Herder, E. J. Brunner, W. Rathmann et al., "Elevated levels of the anti-inflammatory interleukin-1 receptor antagonist precede the onset of type 2 diabetes: the Whitehall II study," Diabetes Care, vol. 32, no. 3, pp. 421-423, 2009.

[6] I. Manabe, "Chronic inflammation links cardiovascular, metabolic and renal diseases," Circulation Journal, vol. 75, no. 12, pp. 2739-2748, 2011.

[7] C. C. Wu, H. K. Sytwu, and Y. F. Lin, "Cytokines in diabetic nephropathy," Advances in Clinical Chemistry, vol. 56, pp. 5574, 2012.

[8] R. B. Goldberg, "Cytokine and cytokine-like inflammation markers, endothelial dysfunction, and imbalanced coagulation in development of diabetes and its complications," Journal of Clinical Endocrinology and Metabolism, vol. 94, no. 9, pp. 31713182, 2009.

[9] K. Matsumoto, Y. Sera, Y. Ueki, G. Inukai, E. Niiro, and S. Miyake, "Comparison of serum concentrations of soluble adhesion molecules in diabetic microangiopathy and macroangiopathy," Diabetic Medicine, vol. 19, no. 10, pp. 822-826, 2002.

[10] M. F. Lopes-Virella, R. E. Carter, G. E. Gilbert et al., "Risk factors related to inflammation and endothelial dysfunction in the DCCT/EDIC cohort and their relationship with nephropathy and macrovascular complications," Diabetes Care, vol. 31, no. 10, pp. 2006-2012, 2008.

[11] A. S. Astrup, L. Tarnow, L. Pietraszek et al., "Markers of endothelial dysfunction and inflammation in type 1 diabetic patients with or without diabetic nephropathy followed for 10 years: association with mortality and decline of glomerular filtration rate," Diabetes Care, vol. 31, no. 6, pp. 1170-1176, 2008.

[12] C. Heldin, M. Landström, and A. Moustakas, "Mechanism of TGF- $\beta$ signaling to growth arrest, apoptosis, and epithelialmesenchymal transition," Current Opinion in Cell Biology, vol. 21, no. 2, pp. 166-176, 2009.

[13] J. Massagué and D. Wotton, "Transcriptional control by the TGF- $\beta$ /Smad signaling system," EMBO Journal, vol. 19 , no. 8, pp. 1745-1754, 2000.

[14] W. Wang, V. Koka, and H. Y. Lan, "Transforming growth factor$\beta$ and Smad signalling in kidney diseases," Nephrology, vol. 10, no. 1, pp. 48-56, 2005.

[15] Y. Qian, E. Feldman, S. Pennathur, M. Kretzler, and F. C. Brosius, "From fibrosis to sclerosis: mechanisms of glomerulosclerosis in diabetic nephropathy," Diabetes, vol. 57, no. 6, pp. 1439-1445, 2008.

[16] T. Yamamoto, T. Nakamura, N. A. Noble, E. Ruoslahti, and W. A. Border, "Expression of transforming growth factor $\beta$ is elevated in human and experimental diabetic nephropathy," Proceedings of the National Academy of Sciences of the United States of America, vol. 90, no. 5, pp. 1814-1818, 1993.

[17] Y. Qian, E. Feldman, S. Pennathur, M. Kretzler, and F. C. Brosius, "From fibrosis to sclerosis: mechanisms of glomerulosclerosis in diabetic nephropathy," Diabetes, vol. 57, no. 6, pp. 1439-1445, 2008.

[18] K. Sharma and F. N. Ziyadeh, "Hyperglycemia and diabetic kidney disease: the case for transforming growth factor- $\beta$ as a key mediator," Diabetes, vol. 44, no. 10, pp. 1139-1146, 1995.

[19] W. A. Border, N. A. Noble, T. Yamamoto et al., "Natural inhibitor of transforming growth factor- $\beta$ protects against scarring in experimental kidney disease," Nature, vol. 360, no. 6402, pp. 361-364, 1992.

[20] E. W. R. Rivarola, M. Moyses-Neto, M. Dantas, C. G. DaSilva, R. Volpini, and T. M. Coimbra, "Transforming growth factor $\beta$ activity in urine of patients with type 2 diabetes and diabetic nephropathy," Brazilian Journal of Medical and Biological Research, vol. 32, no. 12, pp. 1525-1528, 1999. 
[21] D. Mucida, Y. Park, G. Kim et al., "Reciprocal TH17 and regulatory $\mathrm{T}$ cell differentiation mediated by retinoic acid," Science, vol. 317, no. 5835, pp. 256-260, 2007.

[22] M. Veldhoen, R. J. Hocking, C. J. Atkins, R. M. Locksley, and B. Stockinger, "TGF $\beta$ in the context of an inflammatory cytokine milieu supports de novo differentiation of IL-17-producing T cells," Immunity, vol. 24, no. 2, pp. 179-189, 2006.

[23] K. Okamoto, Y. Iwai, M. Oh-Hora et al., "I $\kappa \mathrm{B} \zeta$ regulates TH 17 development by cooperating with ROR nuclear receptors," Nature, vol. 464, no. 7293, pp. 1381-1385, 2010.

[24] W. A. Border, S. Okuda, L. R. Languino, M. B. Sporn, and E. Ruoslahti, "Suppression of experimental glomerulonephritis by antiserum against transforming growth factor $\beta 1$," Nature, vol. 346, no. 6282, pp. 371-374, 1990.

[25] Y. Isaka, Y. Fujiwara, N. Ueda, Y. Kaneda, T. Kamada, and E. Imai, "Glomerulosclerosis induced by in vivo transfection of transforming growth factor- $\beta$ or platelet-derived growth factor gene into the rat kidney," Journal of Clinical Investigation, vol. 92, no. 6, pp. 2597-2601, 1993.

[26] Z. I. Niemir, H. Stein, I. L. Noronha et al., "PDGF and TGF$\beta$ contribute to the natural course of human IgA glomerulonephritis," Kidney International, vol. 48, no. 5, pp. 1530-1541, 1995.

[27] N. Perico and G. Remuzzi, "Inhibition of TGF- $\beta$ expression: a novel role for thiazolidinediones to implement renoprotection in diabetes," Kidney International, vol. 72, no. 12, pp. 1419-1421, 2007.

[28] S. Ohtomo, Y. Izuhara, S. Takizawa et al., "Thiazolidinediones provide better renoprotection than insulin in an obese, hypertensive type II diabetic rat model," Kidney International, vol. 72, no. 12, pp. 1512-1519, 2007.

[29] S. Ohga, K. Shikata, K. Yozai et al., "Thiazolidinedione ameliorates renal injury in experimental diabetic rats through antiinflammatory effects mediated by inhibition of NF- $\kappa \mathrm{B}$ activation," The American Journal of Physiology-Renal Physiology, vol. 292, no. 4, pp. F1141-F1150, 2007.

[30] D. A. Lawrence, "Transforming growth factor- $\beta$ : an overview," Kidney International Supplements, no. 49, pp. S19-S23, 1995.

[31] Y. Watanabe, A. Kinoshita, T. Yamada et al., "A catalog of 106 single-nucleotide polymorphisms (SNPs) and 11 other types of variations in genes for transforming growth factor- $\beta 1$ (TGF- $\beta 1$ ) and its signaling pathway," Journal of Human Genetics, vol. 47, no. 9, pp. 478-483, 2002.

[32] R. Derynck, L. Rhee, E. Y. Chen, and A. van Tilburg, "Intronexon structure of the human transforming growth factor- $\beta$ precursor gene," Nucleic Acids Research, vol. 15, no. 7, pp. 31883189, 1987.

[33] T. S. Ahluwalia, M. Khullar, M. Ahuja et al., "Common variants of inflammatory cytokine genes are associated with risk of nephropathy in type 2 diabetes among Asian Indians," PLoS ONE, vol. 4, no. 4, Article ID e5168, 2009.

[34] M. Buraczynska, I. Baranowicz-Gaszczyk, E. Borowicz, and A. Ksiazek, "TGF- $\beta 1$ and TSC-22 gene polymorphisms and susceptibility to microvascular complications in type 2 diabetes," Nephron-Physiology, vol. 106, no. 4, pp. p69-p75, 2007.

[35] Y. Akai, H. Sato, H. Ozaki, M. Iwano, Y. Dohi, and M. Kanauchi, "Association of transforming growth factor- $\beta 1$ T29C polymorphism with the progression of diabetic nephropathy," The American Journal of Kidney Diseases, vol. 38, no. 4, pp. S182S185, 2001.

[36] T. Y. H. Wong, P. Poon, K. M. Chow, C. C. Szeto, M. K. Cheung, and P. K. T. Li, "Association of transforming growth factor- $\beta$
(TGF- $\beta$ ) T869C (Leu 10Pro) gene polymorphisms with type 2 diabetic nephropathy in Chinese," Kidney International, vol. 63, no. 5, pp. 1831-1835, 2003.

[37] M. Buraczynska, I. Baranowicz-Gaszczyk, E. Borowicz, and A. Ksiazek, "TGF- $\beta 1$ and TSC-22 gene polymorphisms and susceptibility to microvascular complications in type 2 diabetes," Nephron-Physiology, vol. 106, no. 4, pp. p69-p75, 2007.

[38] X. Mou, W. H. Liu, D. Y. Zhou et al., "Association of Chinese medicine constitution susceptibility to diabetic nephropathy and transforming growth factor- $\beta 1$ (T869C) gene polymorphism," Chinese Journal of Integrative Medicine, vol. 17, no. 9, pp. 680-684, 2011.

[39] N. Babel, L. Gabdrakhmanova, M. H. Hammer et al., "Predictive value of cytokine gene polymorphisms for the development of end-stage renal disease," Journal of Nephrology, vol. 19, no. 6, pp. 802-807, 2006.

[40] A. Valladares-Salgado, J. Angeles-MartÍnez, M. Rosas et al., "Association of polymorphisms within the transforming growth factor- $\beta 1$ gene with diabetic nephropathy and serum cholesterol and triglyceride concentrations," Nephrology, vol. 15, no. 6, pp. 644-648, 2010.

[41] M. Minami, N. Kume, H. Kataoka et al., "Transforming growth factor- $\beta 1$ increases the expression of lectin-like oxidized lowdensity lipoprotein receptor-1," Biochemical and Biophysical Research Communications, vol. 272, no. 2, pp. 357-361, 2000.

[42] X. Zhou, T. P. Johnston, D. Johansson et al., "Hypercholesterolemia leads to elevated TGF- $\beta 1$ activity and T helper 3dependent autoimmune responses in atherosclerotic mice," Atherosclerosis, vol. 204, no. 2, pp. 381-387, 2009.

[43] T. S. Ahluwalia, M. Khullar, M. Ahuja et al., "Common variants of inflammatory cytokine genes are associated with risk of nephropathy in type 2 diabetes among Asian Indians," PLoS ONE, vol. 4, no. 4, Article ID e5168, 2009.

[44] P. Prasad, A. K. Tiwari, K. M. P. Kumar et al., "Association of TGF $\beta 1$, TNF $\alpha$, CCR2 and CCR5 gene polymorphisms in type2 diabetes and renal insufficiency among Asian Indians," $B M C$ Medical Genetics, vol. 8, article 20, 2007.

[45] H. Jia, L. Yu, B. Gao, and Q. Ji, "Association between the T869C polymorphism of transforming growth factor- $\beta 1$ and diabetic nephropathy: a meta-analysis," Endocrine, vol. 40, no. 3, pp. 372-378, 2011.

[46] M. Murphy, F. Hickey, and C. Godson, "IHG-1 amplifies TGF$\beta 1$ signalling and mitochondrial biogenesis and is increased in diabetic kidney disease," Current Opinion in Nephrology and Hypertension, vol. 22, no. 1, pp. 77-84, 2013.

[47] X. Cheng, W. Gao, Y. Dang et al., "Both ERK/MAPK and TGF$\beta /$ Smad signaling pathways play a role in the kidney fibrosis of diabetic mice accelerated by blood glucose fluctuation," Journal of Diabetes Research, vol. 2013, Article ID 463740, 8 pages, 2013. 


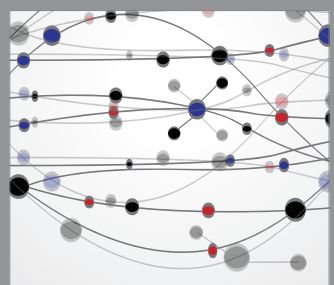

The Scientific World Journal
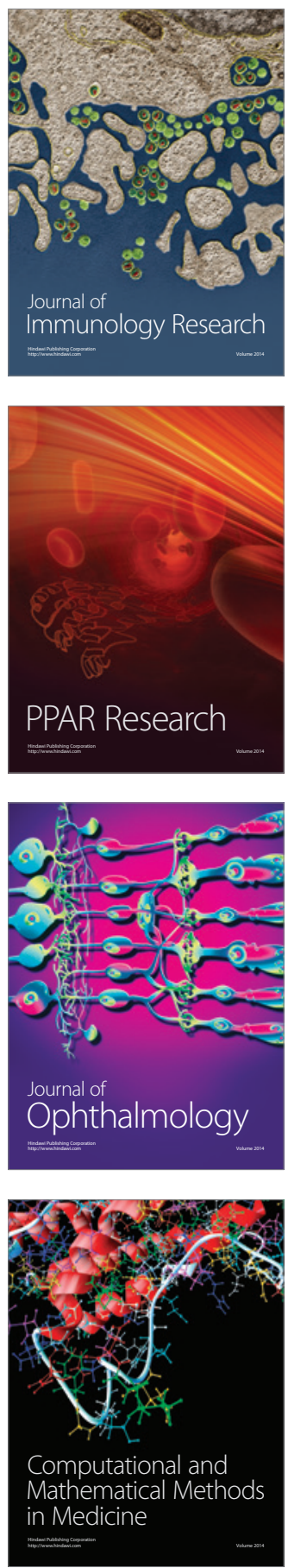

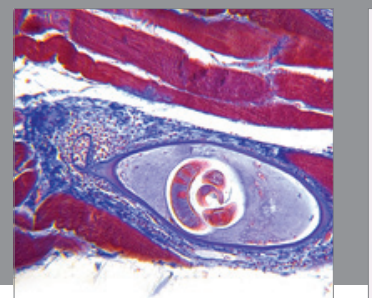

Gastroenterology

Research and Practice
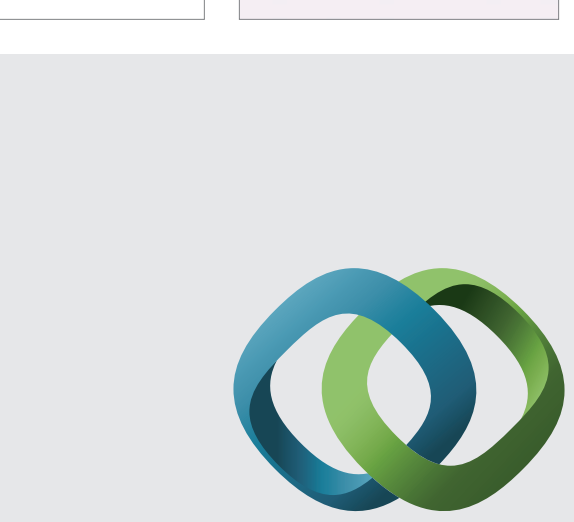

\section{Hindawi}

Submit your manuscripts at

http://www.hindawi.com
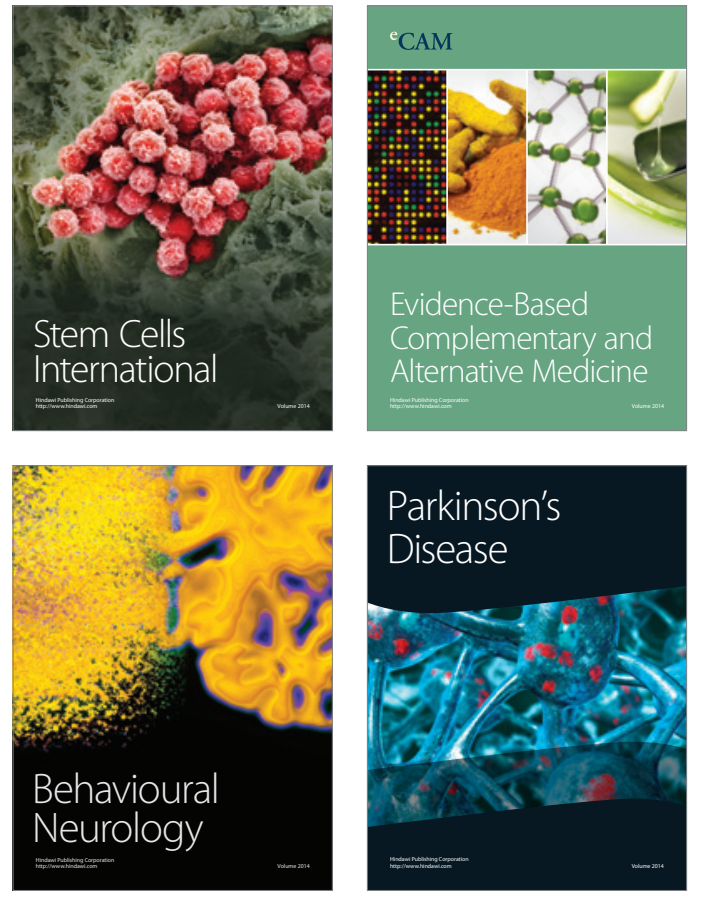
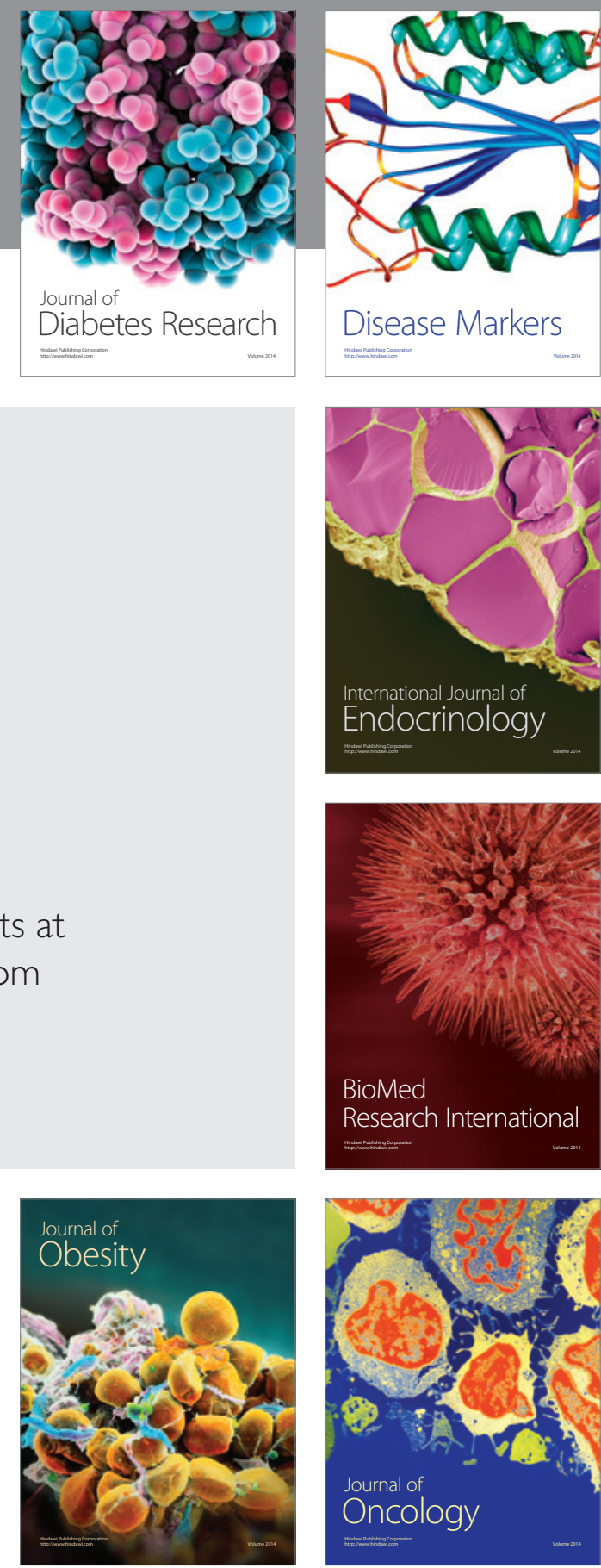

Disease Markers
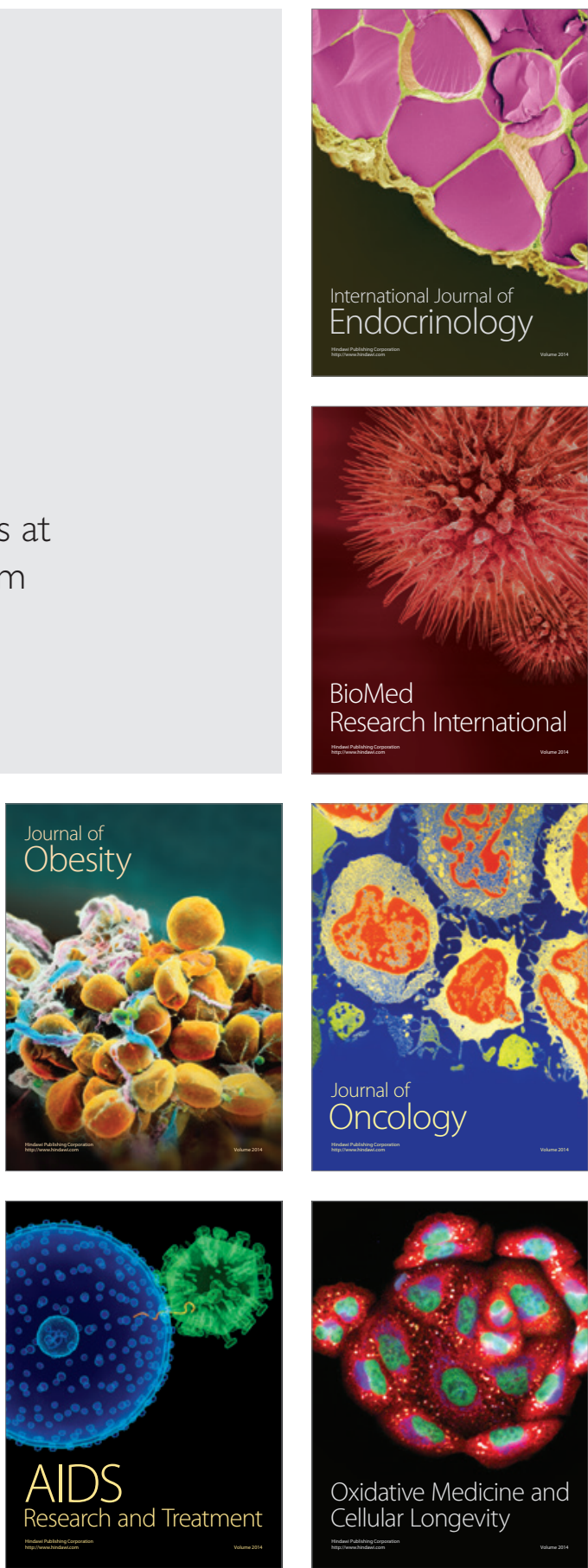